Dementia

and Geriatric

Cognitive Disorders
Dement Geriatr Cogn Disord 2006;22:306-311

DOI: $\underline{10.1159 / 000095303}$
Accepted: September 24, 2005

Published online: August 23, 2006

\title{
Multiple Cognitive Deficits in Amnestic Mild Cognitive Impairment
}

\author{
Joel H. Kramer ${ }^{a}$ Adam Nelson $^{a}$ Julene K. Johnson ${ }^{a}$ Kristine Yaffe ${ }^{a, b}$ \\ Shenly Glenn ${ }^{a}$ Howard J. Rosen ${ }^{a}$ Bruce L. Miller ${ }^{a}$

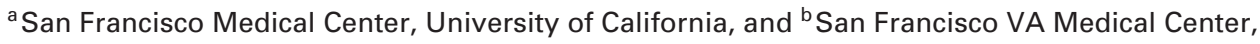 \\ San Francisco, Calif., USA
}

\section{Key Words}

Amnestic mild cognitive impairment · Alzheimer's disease $\cdot$ Executive function $\cdot$ Fluency

\begin{abstract}
Objective: To determine if more widespread cognitive deficits are present in a narrowly defined group of patients with the amnestic form of mild cognitive impairment (MCl). Methods: From a larger sample of patients clinically diagnosed as meeting the criteria of Petersen et al. for amnestic $\mathrm{MCl}$, we selected 22 subjects who had Clinical Dementia Rating scores of zero on all domains besides memory and orientation. These $\mathrm{MCl}$ subjects with presumably isolated memory impairments were compared to 35 age-matched normal controls and 33 very mild Alzheimer's disease $(A D)$ patients on a battery of neuropsychological tests. Result: In addition to the expected deficits in episodic memory, the amnestic $\mathrm{MCl}$ group performed less well than the controls but better than the AD group on design fluency, category fluency, a set shifting task and the Stroop interference condition. Over half the amnestic $\mathrm{MCl}$ group (vs. none of the normal controls) scored at least 1 standard deviation below control means on 4 or more of the nonmemory cognitive
\end{abstract}

tasks. Conclusions: Isolated memory impairment may be fairly uncommon in clinically diagnosed amnestic $\mathrm{MCl}$ patients, even when the criteria for amnestic $\mathrm{MCl}$ are fairly narrow. Additional cognitive impairments are likely to include fluency and executive functioning. These more diffuse deficits argue for comprehensive cognitive assessments, even when the patient and family are reporting only memory decline, and are consistent with the increase in attention paid to the heterogeneity of $\mathrm{MCl}$.

Copyright $(2006$ S. Karger AG, Basel

Mild cognitive impairment (MCI) lies intermediate between normal aging and dementia [1]. Despite variability in how MCI is operationally defined, elderly individuals with $\mathrm{MCI}$ are consistently shown to have a higher rate of progression to dementia [2-5].

Although subtypes of MCI have been reported [6-8], the amnestic form is most frequently studied, and criteria for MCI typically emphasize memory deficits. Among the most widely cited criteria for MCI are those proposed by Petersen et al. [9], which require memory complaint, normal activities of daily living, normal general cognitive function, abnormal memory for age and absence of dementia. How normal general cognitive function is deter-

\section{KARGER \\ Fax +4161306 1234 E-Mailkarger@karger.ch} www.karger.com
(C) 2006 S. Karger AG, Basel $1420-8008 / 06 / 0224-0306 \$ 23.50 / 0$

Accessible online at: www.karger.com/dem
Joel Kramer, PsyD

Memory and Aging Center, UCSF Medical Center

Box 1207, 350 Parnassus, Suite 706

San Francisco, CA 94143 (USA)

Tel. +1 415476 7561, Fax +1 415476 4800, E-Mail kramer@itsa.ucsf.edu 
mined, however, varies across studies and includes clinical impression, Mini Mental State Examination (MMSE), Clinical Dementia Rating Scale (CDR) [10] and cognitive testing. Petersen et al. [9], for example, used the intelligence quotient as a measure of general cognitive abilities and reported no differences between their MCI and normal control cohorts.

One important unanswered question is whether amnestic MCI patients are, in fact, free of the widespread cognitive deficits characteristic of early Alzheimer's disease (AD). Global measures of cognition such as the intelligence quotient and memory-oriented tasks like the MMSE can obscure focal deficits in domains such as language, visuospatial ability, working memory and executive functioning. In addition, even CDR scores of 0 or 0.5 do not rule out deficits in judgment or declining participation in community affairs. In the study of Petersen et al. [9], for example, their MCI cohort had a mean sum of box scores on the CDR of 1.5, implying that despite average intelligence quotient scores, some nonmemory domains of functioning may have been abnormal.

Studies of amnestic MCI typically define their cohort somewhat broadly, either on the basis of clinical complaint or a CDR score of 0.5. This leaves unanswered the clinically relevant question of whether subtle nonmemory deficits are going undetected. While some recent studies have suggested that nonmemory deficits are often present $[2,11]$, their subjects were drawn from a general population of MCI patients without any specific methods to select subjects with isolated memory impairment. The goal of the present study was to determine whether MCI patients who present with 'isolated' memory deficits have more diffuse cognitive impairment. To accomplish this, we began with a cohort of patients with CDR scores of 0.5 who met the criteria of Petersen et al. [9] for amnestic MCI. To create a sample with presumed isolated memory impairment, we then excluded cases with more widespread functional decline by requiring that all nonmemory domains assessed by the CDR were zero. This approach enabled the creation of a cohort that, per an informant, had no nonmemory functional deficits.

\section{Methods}

\section{Subjects}

The initial sample of subjects consisted of 86 patients diagnosed as having MCI at the UCSF Memory and Aging Center with a CDR total score of less than 1.0. The diagnostic evaluation consisted of a complete history and physical examination, interviews with an informant, completion of the CDR and any appropriate laboratory and neuroimaging assessments to rule out identifiable causes for cognitive change. The CDR was completed by a clinic nurse who completed the Brief Training and Reliability Protocol offered by the Washington University Alzheimer's Disease Research Center. Subjects also received bedside neurocognitive testing blind to clinical diagnosis. The diagnosis was made by consensus conference where all clinicians who participated in the evaluation were present. A diagnosis of amnestic MCI was made using the criteria of Petersen et al. [9], which require memory complaint, typically corroborated by an informant, normal activities of daily living, normal general cognitive function, abnormal memory for age and absence of dementia. The determination of amnestic MCI was based on clinical grounds, and, as in the original paper of Petersen et al. [9], psychometric cut-off scores were not used. Individual CDR domain scores were then examined to select only those cases that were rated as a zero on the CDR domains of 'judgment', 'community affairs', 'home and hobbies' and 'personal care'. Twenty-two cases met this criterion. Of these 22 cases, 20 were rated as 0.5 on 'memory' and 2 were rated 0 . Only 1 of these 22 subjects received a 0.5 for 'orientation'; the other 21 cases had ratings of 0 on orientation. One subject was rated as zero for memory and orientation.

The cognitive performance of our amnestic MCI was compared to two control groups, normal controls and patients with very mild AD. Both control groups were drawn from the Memory and Aging Center. The mild AD control group consisted of 33 patients who met NINCDS-ADRDA criteria for probable AD, had CDR total scores of 1 or less and MMSE scores greater than 23. The normal control group consisted of 35 normal elderly subjects over the age of 64 years recruited for research by the Memory and Aging Center. Inclusion into the normal control cohort required a CDR of 0 , absence of significant cognitive complaints from the subject and informant, and a normal neurological examination.

Subjects in all three groups underwent laboratory testing and neuroimaging to rule out other medical conditions that can cause cognitive impairment. Exclusion criteria included abrupt onset of cognitive impairment, evidence for significant CNS effects of cerebrovascular disease, specifically the presence of severe periventricular white matter disease, large confluent white matter lesions, or grade 4 white matter lesions as described by Scheltens et al. [12], more than 1 lacunar infarct, any cortical strokes, CNS lesions on neuroimaging, including parenchymal mass lesion or extra-axial mass lesion with visible compression of the brain parenchyma, communicating or noncommunicating hydrocephalus or evidence of demyelinating disease, focal inflammatory or infectious disease or recent or remote intracerebral hemorrhage, substance abuse (including alcohol) or dependence, history of head trauma with loss of consciousness greater than $30 \mathrm{~min}$, metabolic or systemic abnormalities with possible impact on cognition including uncorrected $\mathrm{B}_{12}$ deficiency, uncorrected hypothyroidism, renal insufficiency requiring dialysis, significant liver dysfunction (with deficiency in synthetic function), respiratory insufficiency requiring oxygen or HIV positive status.

Demographic data are summarized in table 1 . There were no group differences in age or education.

\section{Neuropsychological Assessment}

All subjects completed a brief neuropsychological screening designed to quickly and reliably assess episodic memory, working memory, executive functioning, fluency, naming, spatial ability and abstract reasoning [13]. The screening typically required 
Table 1. Mean demographic and neuropsychological data in the normal control (NC), MCI and mild AD groups

\begin{tabular}{|c|c|c|c|c|c|c|c|c|}
\hline & \multicolumn{2}{|l|}{$\mathrm{NC}$} & \multicolumn{2}{|c|}{ Amnestic MCI } & \multicolumn{2}{|l|}{$\mathrm{AD}$} & \multirow{2}{*}{$\begin{array}{l}\text { MCI versus } \\
\mathrm{NC}\end{array}$} & \multirow{2}{*}{$\begin{array}{l}\text { MC versus } \\
\mathrm{AD}\end{array}$} \\
\hline & mean & SD & mean & SD & mean & SD & & \\
\hline Age & 73.0 & 5.3 & 75.0 & 6.1 & 73.4 & 9.2 & n.s. & n.s. \\
\hline Education & 16.6 & 2.8 & 16.5 & 3.2 & 15.7 & 3.3 & n.s. & n.s. \\
\hline MMSE & 29.5 & 0.8 & 28.5 & 1.5 & 25.2 & 1.3 & $<0.05$ & $<0.001$ \\
\hline Delayed verbal memory & 7.3 & 1.8 & 4.5 & 2.8 & 1.6 & 1.9 & $<0.001$ & $<0.001$ \\
\hline Delayed visual memory & 11.4 & 3.0 & 8.6 & 4.6 & 3.6 & 3.7 & $<0.05$ & $<0.001$ \\
\hline Digits backward & 5.0 & 1.2 & 4.7 & 1.0 & 4.0 & 1.0 & n.s. & n.s. \\
\hline Modified trails & 3.3 & 0.5 & 2.7 & 0.9 & 2.0 & 0.8 & $<0.005$ & $<0.005$ \\
\hline Stroop & 49.7 & 9.7 & 38.8 & 10.4 & 19.9 & 10.2 & $<0.001$ & $<0.001$ \\
\hline Design fluency & 10.1 & 2.9 & 8.0 & 2.9 & 5.3 & 2.2 & $<0.05$ & $<0.05$ \\
\hline d-Word generation & 15.3 & 5.7 & 13.3 & 5.4 & 10.6 & 4.4 & n.s. & n.s. \\
\hline Animal fluency & 21.5 & 5.9 & 15.6 & 5.0 & 9.6 & 5.1 & $<0.001$ & $<0.001$ \\
\hline Boston naming & 14.5 & 0.8 & 13.5 & 1.3 & 11.4 & 3.3 & n.s. & $<0.05$ \\
\hline Modified Rey copy & 15.4 & 1.4 & 15.2 & 1.7 & 14.2 & 2.8 & n.s. & n.s. \\
\hline Abstract reasoning & 4.8 & 1.0 & 4.5 & 1.3 & 2.9 & 1.5 & n.s. & $<0.001$ \\
\hline GDS & 4.3 & 3.7 & 6.3 & 3.9 & 7.7 & 6.7 & n.s. & n.s. \\
\hline
\end{tabular}

n.s. = Not significant; GDS $=$ Geriatric Depression Scale.

45 min to complete. Verbal episodic memory was evaluated with the California Verbal Learning Test - Short Form [14]. Visual memory was assessed by having subjects draw from memory a geometric figure they had copied 10 min earlier. Recall of the figure was scored on a 17 -point scale. Working memory was measured using backward digit span length. Executive functioning tasks included cognitive flexibility and response inhibition. Cognitive flexibility was assessed using a modification of the Trail-Making Test that required subjects to serially alternate between numbers and days of the week. To adjust for the fact that some subjects did not complete the task within the 2-min time limit, the dependent measure was the number of correct connections made per minute (log transformed to normalize the distribution). Response inhibition was assessed by the interference condition of the Stroop test; their final score was the total number of correct responses in $60 \mathrm{~s}$. Verbal fluency was number of words beginning with the letter ' $\mathrm{d}$ ' in $1 \mathrm{~min}$ and number of animals in $1 \mathrm{~min}$. Design fluency was assessed using condition 1 of the Delis-Kaplan Executive Functioning System Design Fluency subtest that required subjects to generate novel designs with 4 lines. Confrontation naming was assessed with a 15 item short form of the Boston Naming Test. Spatial ability was assessed by having subjects copy a complex geometric figure [13]. Interpretation of proverbs and determining conceptual similarities between word pairs served as the measure of abstract reasoning.

\section{Statistical Procedures}

Group differences on the cognitive measures were evaluated using one-way ANOVA with Tukey's post hoc contrasts, with the contrasts of primary interest being MCI versus control and MCI versus $\mathrm{AD}$.

\section{Results}

Mean scores on the neuropsychological test for the $\mathrm{AD}, \mathrm{MCI}$ and normal control groups are listed in table 1. There were no group differences in age or education. On the MMSE, the MCI group's mean score of 28.5 (range = 26-30) was well within normal limits but below that of the normal controls and higher than that of the AD patients. As expected, the MCI group also performed less well than the control group but better than the AD group on measures of delayed verbal and visual memory.

Significant group differences were found for all of the neuropsychological variables except design copying, with the $\mathrm{AD}$ group performing less well than controls on all measures.

There were significant differences between the MCI and normal control groups on several fluency and executive tasks. The MCI group performed less well than the normal control group but better than the AD group on design fluency, category fluency, modified trails and the Stroop interference condition. The MCI subjects performed comparably to both the normal controls and AD subjects on d-word generation. There were no differences between the MCI subjects and normal controls on Boston naming, backward digit span and abstract reasoning; both groups obtained higher scores than the $\mathrm{AD}$ group. 
Table 2. Number of MCI, AD and normal subjects scoring in the impaired range

\begin{tabular}{llll}
\hline $\begin{array}{l}\text { Number of nonmemory tests } \\
\text { in the impaired range }\end{array}$ & $\begin{array}{l}\text { Amnestic } \\
\text { MCI }\end{array}$ & AD & $\begin{array}{l}\text { Normal } \\
\text { controls }\end{array}$ \\
\hline $\begin{array}{l}\text { Using cut-off of 1 standard deviation below the mean } \\
0\end{array}$ & 1 & 0 & 16 \\
1 & 6 & 1 & 7 \\
2 & 0 & 1 & 7 \\
3 & 3 & 3 & 5 \\
4 & 8 & 4 & 0 \\
$5+$ & 4 & 24 & 0 \\
\hline Using cut-off of 1.5 standard deviations below the mean \\
0 & 6 & 1 & 21 \\
1 & 4 & 2 & 10 \\
2 & 3 & 3 & 2 \\
3 & 5 & 3 & 2 \\
4 & 2 & 5 & 0 \\
$5+$ & 2 & 19 & 0 \\
\hline
\end{tabular}

To rule out the possibility that MCI subjects did less well on the fluency tasks because of poor memory (and a related increase in perseverative responses), the analyses for category and design fluency were repeated using the total number of responses (correct + perseverative). Results were unchanged.

We also tabulated the number of amnestic MCI subjects who performed in the impaired range on the nonmemory tasks (table 2). Two different cut-offs for impairment were used: worse than 1 standard deviation below the mean and worse than 1.5 standard deviations below the mean. When 1 standard deviation below the mean was used as the cut-off, 4 of the 22 MCI subjects performed below this threshold on 5 of the cognitive tasks, 8 subjects scored below the threshold on 4 tasks, 3 subjects scored below this threshold on 3 tasks, and 6 subjects scored below the threshold on 2 tasks. Only 1 of the amnestic MCI subjects scored within a standard deviation of the normal control mean on all nonmemory cognitive tasks. In contrast, only 5 of the normal controls had 3 or more performances below the 1 standard deviation cutoff. When the more conservative 1.5 standard deviation cut-off was used, 4 MCI subjects scored in the impaired range of 4 or more nonmemory tests, whereas none of the normal controls did.

\section{Discussion}

One of the major findings of the present study is that non-memory deficits were found in a narrowly defined group of amnestic MCI subjects. To be included in this cohort, scores on the nonmemory domains of the CDR had to be zero, implying intact judgment and normal functioning at home and in the community. Nonetheless, these subjects scored significantly lower than normal agematched controls on 4 of the 9 nonmemory cognitive tasks, performing less well on measures of executive functioning, category fluency and design fluency. Over half the amnestic MCI group scored at least 1 standard deviation below control means on at least 4 of the nonmemory cognitive tasks.

This study further demonstrates the low prevalence of MCI cases with isolated memory deficits. We began with a cohort of $86 \mathrm{MCI}$ subjects who met the criteria of Petersen et al. [9] for amnestic MCI. Of these 86 cases, 64 had evidence for dysfunction in nonmemory domains on the CDR. Only 22 cases, approximately $25 \%$, had CDR scores of zero in all the nonmemory domains. In addition, only 1 of the 22 amnestic-MCI subjects in our final sample scored within 1 standard deviation of the mean of our normal controls on all of the nonmemory cognitive tasks, whereas 16 of the normal controls performed at this level. This low incidence of isolated memory impairment is consistent with other reports in the literature. Lopez et al. [8], for example, reported on 130 participants from the Cardiovascular Health Study with MCI. Using neuropsychological test scores to classify subjects, they found that about $28 \%$ had isolated memory impairment, whereas the remaining $72 \%$ had deterioration in at least 1 nonmemory cognitive domain. This percentage is highly concordant with our CDR data that showed that only $25 \%$ of clinically diagnosed amnestic MCI cases had isolated memory dysfunction. A study of retirement community residents also showed that $25-35 \%$ of memory-impaired subjects also had executive impairment [15].

Morris et al. [16] have argued that MCI generally represents early-stage $\mathrm{AD}$ and that MCI patients almost always have the neuropathological features of $\operatorname{AD}[17,18]$. If this is the case, we might expect incipient cognitive changes in an MCI cohort that parallel those found in early AD. Neuropsychological studies demonstrate that in addition to memory, cognitive tasks particularly sensitive to early AD include category fluency and executive functioning [19-23]. Other studies of patients with questionable dementia also reported diminished category fluency [24]. Our findings of fluency and executive deficits

Dement Geriatr Cogn Disord 2006;22:306-311 
in $\mathrm{MCI}$ are consistent with the view that amnestic MCI frequently represents a prodrome of $\mathrm{AD}$. The more widespread cognitive difficulties in our amnestic MCI cohort suggest that they are at greater risk for conversion to dementia. Among nondemented elderly individuals, the risk of dementia significantly increased among patients with clear cognitive impairments beyond memory loss [25-27] whereas memory loss alone progressed to dementia less often over a 2-year period [26].

General cognition in our amnestic MCI subjects was clinically determined to be normal. Although the mean MMSE score in the amnestic MCI group was lower than that of the normal controls, a mean MMSE of 28.5 is not only entirely within normal limits, it is actually higher than the mean MMSE score (mean $=28.3$ ) of the normal controls used in the original study of Petersen et al. [9]. The MCI group's MMSE was also higher than the 24 that several groups use as a cut-off [28] for indicating global impairment, and none of the subjects in our sample had an MMSE below 26. Normal general functioning was also reflected in our subjects' CDR scores, where the mean sum of box scores was quite low.

One of the ongoing issues in MCI research is how to best define constructs like amnestic MCI and normal general cognitive function $[28,29]$. The issue of diagnostic criteria is critically important, since how subjects are categorized can shift with even minor changes in the criteria. Some studies take a psychometric approach to both constructs. Abnormal memory or other cognitive ability is often defined as 1.5 standard deviations below the mean or below a particular cut-off score on a neuropsychological test $[8,30]$. Alternately, diagnosis of amnestic MCI has been based on patient complaints [9] or clinician impression [31]. Both approaches have their strengths and weaknesses. Clinical impression is often difficult to operationalize, and establishing reliability across groups of clinicians is challenging. On the other hand, relying on psychometric tests alone can also be problematic. Performance on neuropsychological tests is affected by many factors, including education, age, cultural background, sensorimotor deficits and illnesses other than AD [1]. In addition, many standardized neuropsychological tests have small normative samples of elderly subjects that may not be representative of the population, and skewed distributions can affect the number of cases falling outside a given standard deviation cut-off. Finally, there is no consensus on which tests or what types of scores (e.g. immediate recall, delayed recall, savings scores, recognition) should be used. The approach taken in the present study was to use a combination of clinical impression and the CDR, a standardized metric widely used for staging dementia severity, reserving the neuropsychological test scores for outcome measures. One of the advantages of this approach is that it more closely parallels what occurs in a clinic setting where formal neuropsychological testing may not be available.

This study relied on the CDR, an informant-based measure, for identifying MCI cases with isolated memory deficits. The quality of these data, however, depends on the insightfulness and accuracy of the informant. It remains possible that informants were not aware of extant nonmemory deficits, although collateral sources tend to be fairly accurate when describing the cognitive impairments in patients with even very early dementia [32].

In sum, the majority of patients that met fairly narrow criteria for amnestic MCI in a memory disorders clinic had evidence for more widespread cognitive deficits on formal neuropsychological testing. The pattern of neuropsychological deficits, characterized by poor episodic memory and difficulties with executive and fluency tasks, parallels those found in mild AD. These findings have several important clinical implications. Terms like 'MCI' and 'amnestic MCI' are not consistently defined, blurring their prognostic value and making these important patient groups more difficult to study. Our data suggest that amnestic MCI patients may be a heterogeneous group. Greater evolution of diagnostic criteria is needed, particularly as nonamnestic variants of MCI are more extensively studied. Finally, clinicians should consider the possibility that patients complaining of isolated memory changes may in fact have more diffuse cognitive difficulties suggestive of a neurodegenerative disease and target these patients for early interventions.

\section{Acknowledgements}

This work was supported by the National Institute on Aging grants AG10129, P50-AG05142 and AG16570, the State of California Alzheimer's Disease Research Center of California grant 01154-20 and the Hillblom Foundation grant 2002/2F. 


\section{References}

1 Petersen RC, Doody R, Kurz A, Mohs RC, Morris JC, Rabins PV, et al: Current concepts in mild cognitive impairment. Arch Neurol 2001;58:1985-1992.

2 Petersen RC: Mild cognitive impairment as a diagnostic entity. J Intern Med 2004;256:183194.

-3 Amieva H, Letenneur L, Dartigues JF, RouchLeroyer I, Sourgen C, D'Alchee-Biree F, et al: Annual rate and predictors of conversion to dementia in subjects presenting mild cognitive impairment criteria defined according to a population-based study. Dement Geriatr Cogn Disord 2004; 18:87-93.

-4 Ingles JL, Fisk JD, Merry HR, Rockwood K: Five-year outcomes for dementia defined solely by neuropsychological test performance. Neuroepidemiology 2003;22:172-178.

-5 Storandt M, Grant EA, Miller JP, Morris JC: Rates of progression in mild cognitive impairment and early Alzheimer's disease. Neurology 2002;59:1034-1041.

-6 de Mendonca A, Guerreiro M, Ribeiro F, Mendes T, Garcia C: Mild cognitive impairment: focus on diagnosis. J Mol Neurosci 2004; 23:143-148.

7 Dubois B, Albert ML: Amnestic MCI or prodromal Alzheimer's disease? Lancet Neurol 2004:3:246-248.

-8 Lopez OL, Jagust WJ, DeKosky ST, Becker JT, Fitzpatrick A, Dulberg C, et al: Prevalence and classification of mild cognitive impairment in the Cardiovascular Health Study Cognition Study. Part 1. Arch Neurol 2003;60:13851389.

-9 Petersen RC, Smith GE, Waring SC, Ivnik RJ, Tangalos EG, Kokmen E: Mild cognitive impairment: clinical characterization and outcome. Arch Neurol 1999:56:303-308.

10 Berg L: Clinical Dementia Rating Scale(CDR). Psychopharmacol Bull 1988;24:637-639.

11 Grundman M, Petersen RC, Ferris SH, Thomas RG, Aisen PS, Bennett DA, et al: Mild cognitive impairment can be distinguished from Alzheimer disease and normal aging for clinical trials. Arch Neurol 2004;61:59-66.
12 Scheltens P, Pasquier F, Weerts JG, Barkhof F, Leys D: Qualitative assessment of cerebral atrophy on MRI: inter- and intra-observer reproducibility in dementia and normal aging. Eur Neurol 1997;37:95-99.

13 Kramer JH, Jurik J, Sha SJ, Rankin KP, Rosen HJ, Johnson JK, et al: Distinctive neuropsychological patterns in frontotemporal dementia, semantic dementia, and Alzheimer disease. Cogn Behav Neurol 2003;16:211-218.

14 Delis DC, Kramer JH, Kaplan E, Ober BA: California Verbal Learning Test, ed 2. San Antonio, Psychological Corporation, 2000.

15 Royall DR, Chiodo LK, Polk MJ: Misclassification is likely in the assessment of mild cognitive impairment. Neuroepidemiology 2004; 23:185-191.

-16 Morris JC, Storandt M, Miller JP, McKeel DW, Price JL, Rubin EH, et al: Mild cognitive impairment represents early-stage Alzheimer disease. Arch Neurol 2001;58:397-405.

17 Price JL, Morris JC: Tangles and plaques in nondemented aging and 'preclinical' Alzheimer's disease. Ann Neurol 1999;45:358-368.

18 Price JL, Ko AI, Wade MJ, Tsou SK, McKeel DW, Morris JC: Neuron number in the entorhinal cortex and CA1 in preclinical Alzheimer disease. Arch Neurol 2001;58:1395-1402.

19 De Jager CA, Hogervorst E, Combrinck M, Budge MM: Sensitivity and specificity of neuropsychological tests for mild cognitive impairment, vascular cognitive impairment and Alzheimer's disease. Psychol Med 2003;33: 1039-1050.

20 Ready RE, Ott BR, Grace J, Cahn-Weiner DA: Apathy and executive dysfunction in mild cognitive impairment and Alzheimer disease. Am J Geriatr Psychiatry 2003;11:222-228.

-21 Arnaiz E, Almkvist O: Neuropsychological features of mild cognitive impairment and preclinical Alzheimer's disease. Acta Neurol Scand Suppl 2003;179:34-41.

22 Sgaramella TM, Borgo F, Mondini S, Pasini M, Toso V, Semenza C: Executive deficits appearing in the initial stage of Alzheimer's disease. Brain Cogn 2001;46:264-268.
23 Perry RJ, Hodges JR: Attention and executive deficits in Alzheimer's disease: a critical review. Brain 1999;122:383-404.

24 Vliet EC, Manly J, Tang MX, Marder K, Bell $\mathrm{K}$, Stern Y: The neuropsychological profiles of mild Alzheimer's disease and questionable dementia as compared to age-related cognitive decline. J Int Neuropsychol Soc 2003;9:720732 .

25 Arnaiz E, Almkvist O, Ivnik RJ, Tangalos EG, Wahlund LO, Winblad B, et al: Mild cognitive impairment: a cross-national comparison. J Neurol Neurosurg Psychiatry 2004;75:12751280.

26 Bozoki A, Giordani B, Heidebrink JL, Berent S, Foster NL: Mild cognitive impairments predict dementia in nondemented elderly patients with memory loss. Arch Neurol 2001;58:411416.

27 Guarch J, Marcos T, Salamero M, Blesa R: Neuropsychological markers of dementia in patients with memory complaints. Int J Geriatr Psychiatry 2004;19:352-358.

28 Ganguli M, Dodge HH, Shen C, DeKosky ST: Mild cognitive impairment, amnestic type: an epidemiologic study. Neurology 2004;63:115121.

29 Palmer K, Fratiglioni L, Winblad B: What is mild cognitive impairment? Variations in definitions and evolution of nondemented persons with cognitive impairment. Acta Neurol Scand Suppl 2003;179:14-20.

- 30 Salloway S, Ferris S, Kluger A, Goldman R, Griesing T, Kumar D, et al: Efficacy of donepezil in mild cognitive impairment: a randomized placebo-controlled trial. Neurology 2004; 63:651-657.

- 31 Tierney MC, Szalai JP, Snow WG, Fisher RH: The prediction of Alzheimer disease: the role of patient and informant perceptions of cognitive deficits. Arch Neurol 1996;53:423-427.

- 32 Cacchione PZ, Powlishta KK, Grant EA, Buckles VD, Morris JC: Accuracy of collateral source reports in very mild to mild dementia of the Alzheimer type. J Am Geriatr Soc 2003; 51:819-823. 